\title{
The perfect pattern of moisture transport for precipitation for Arctic sea ice melting
}

Luis Gimeno-Sotelo ${ }^{1}$, Raquel Nieto ${ }^{2}$, Marta Vázquez ${ }^{2}$, Luis Gimeno ${ }^{2}$

${ }^{1}$ Facultade de Matemáticas, Universidade de Santiago de Compostela, 15782 Spain.

5 2Environmental Physics Laboratory (EphysLab), Universidade de Vigo, Ourense, 32004, Spain

Correspondence to: Luis Gimeno (1.gimeno@uvigo.es)

(Supplementary material) 

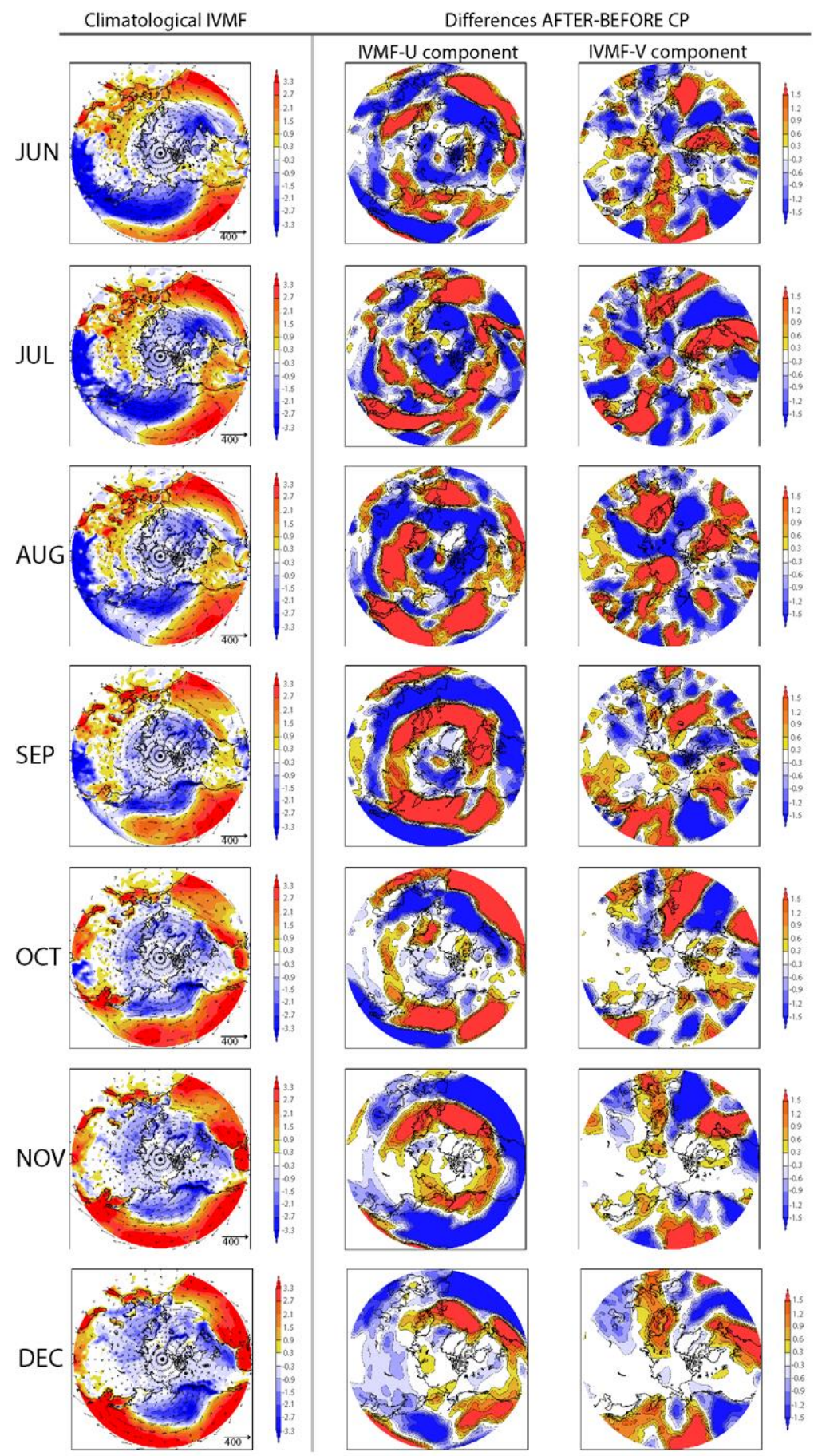

Figure S1. Left) The climatological vertical integrated moisture flux (VIMF) (vector, $\mathrm{kg} / \mathrm{m} / \mathrm{s}$ ) and its divergence (shaded, $\mathrm{mm} / \mathrm{yr}$ ) by month from June to November, middle) the difference between the periods after vs before the $\mathrm{CP}$ for the zonal component of VIMF, 5 and right) as in the middle panel but for the meridional component of VIMF. 

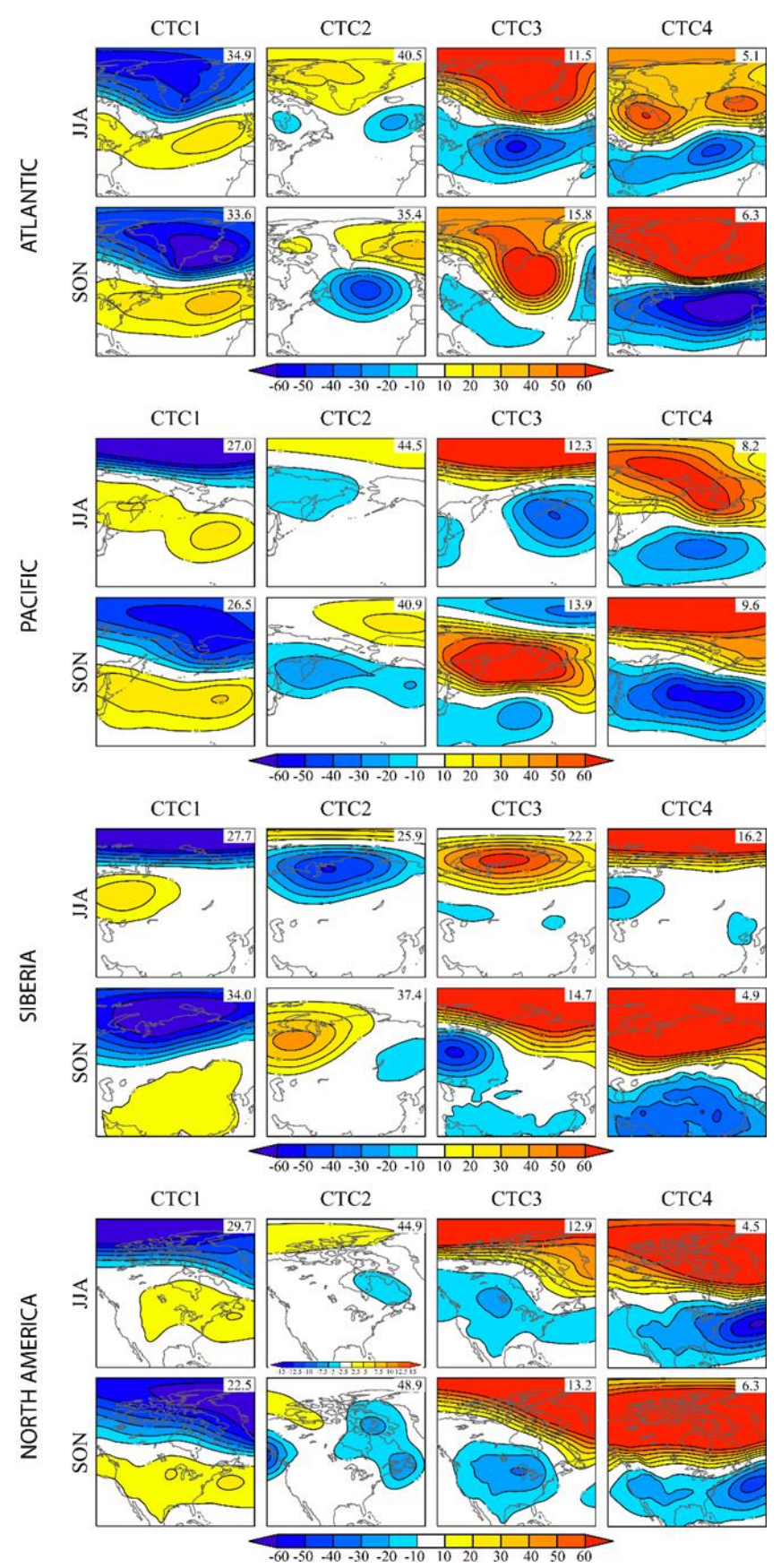

5 Figure S2. Anomalies of geopotential height at $850 \mathrm{hPa}$ (Z850) for the four classes representing types of circulation (classes CTC1 to CTC4), and sector centred on the major source together with the percentage of days grouped in each class 


\begin{tabular}{|c|c|c|c|c|c|c|c|c|}
\hline Month & Mode & $\begin{array}{c}\text { Atlantic } \\
\text { Before/After }\end{array}$ & Mode & $\begin{array}{c}\text { Pacific } \\
\text { Before/After }\end{array}$ & Mode & $\begin{array}{c}\text { Siberian } \\
\text { Before/After }\end{array}$ & Mode & $\begin{array}{c}\text { North America } \\
\text { Before/After }\end{array}$ \\
\hline \multirow[t]{4}{*}{ June } & CTC1 & $3903 / 3552$ & CTC1 & $8961 / 8436$ & CTC1 & $8034 / 7237$ & CTC1 & $10450 / 9289$ \\
\hline & CTC2 & $3626 / 2907$ & CTC2 & $10028 / 9016$ & CTC2 & $8371 / 8530$ & CTC2 & $10140 / 9962$ \\
\hline & CTC3 & $4196 / 2919$ & CTC3 & $8895 / 8463$ & CTC3 & $8348 / 7431$ & СTC3 & $10016 / 10473$ \\
\hline & CTC4 & $3200 / 3276$ & CTC4 & $9918 / 7881$ & CTC4 & $9422 / 8055$ & CTC4 & $8918 / 10746$ \\
\hline \multirow[t]{4}{*}{ July } & CTC1 & $4184 / 3820$ & CTC1 & $1354 / 13634$ & CTC1 & $9122 / 8767$ & CTC1 & $11475 / 12694$ \\
\hline & CTC2 & $4088 / 3550$ & CTC2 & $12983 / 11148$ & CTC2 & $10662 / 8853$ & CTC2 & $11278 / 12008$ \\
\hline & CTC3 & $3980 / 2669$ & CTC3 & $12666 / 12940$ & CTC3 & $9381 / 8200$ & СTC3 & $11563 / 12502$ \\
\hline & CTC4 & $3427 / 3424$ & CTC4 & $12996 / 9504$ & CTC4 & $10224 / 9430$ & CTC4 & $9363 / 11271$ \\
\hline \multirow[t]{4}{*}{ August } & CTC1 & 4018/4659 & CTC1 & $15962 / 14527$ & CTC1 & 9771/8707 & CTC1 & $13017 / 13145$ \\
\hline & CTC2 & $3763 / 4021$ & CTC2 & $14467 / 13273$ & CTC2 & $11564 / 10554$ & CTC2 & $12258 / 12211$ \\
\hline & CTC3 & $3559 / 3530$ & CTC3 & $14714 / 11736$ & CTC3 & $9884 / 9195$ & СТC3 & $12852 / 10896$ \\
\hline & CTC4 & $3731 / 4383$ & CTC4 & $13338 / 13179$ & CTC4 & $10660 / 10466$ & CTC4 & $11864 / 11193$ \\
\hline Month & Mode & $\begin{array}{c}\text { Atlantic } \\
\text { Before/After }\end{array}$ & Mode & $\begin{array}{c}\text { Pacific } \\
\text { Before/After }\end{array}$ & Mode & $\begin{array}{c}\text { Siberian } \\
\text { Before/After }\end{array}$ & Mode & $\begin{array}{c}\text { North America } \\
\text { Before/After }\end{array}$ \\
\hline \multirow[t]{4}{*}{ September } & CTC1 & $4827 / 4955$ & CTC1 & $11648 / 10974$ & CTC1 & $8091 / 9588$ & CTC1 & $11863 / 13180$ \\
\hline & CTC2 & $4877 / 5255$ & CTC2 & $10401 / 10737$ & CTC2 & $8324 / 8306$ & CTC2 & $11865 / 12180$ \\
\hline & CTC3 & $4865 / 5145$ & CTC3 & $12545 / 10308$ & CTC3 & $8479 / 8762$ & СТC3 & $11302 / 11433$ \\
\hline & CTC4 & $4316 / 4655$ & CTC4 & $9760 / 8969$ & CTC4 & $7848 / 9054$ & CTC4 & $11125 / 11095$ \\
\hline \multirow[t]{4}{*}{ October } & CTC1 & $3854 / 4270$ & CTC1 & $8009 / 8121$ & CTC1 & $6703 / 6429$ & CTC1 & $9474 / 8931$ \\
\hline & CTC2 & $4097 / 4545$ & CTC2 & $7792 / 7600$ & CTC2 & $6321 / 6524$ & CTC2 & $9343 / 9328$ \\
\hline & CTC3 & $4132 / 4710$ & CTC3 & $8904 / 7721$ & CTC3 & $6952 / 6728$ & СTC3 & $9541 / 9394$ \\
\hline & CTC4 & $3097 / 4076$ & CTC4 & $7712 / 7157$ & CTC4 & $5785 / 5520$ & CTC4 & $8386 / 8715$ \\
\hline \multirow[t]{4}{*}{ November } & CTC1 & $4182 / 4447$ & CTC1 & $6418 / 6869$ & CTC1 & $4472 / 5181$ & CTC1 & $6484 / 7059$ \\
\hline & CTC2 & $4080 / 4467$ & CTC2 & $6275 / 6214$ & CTC2 & $3859 / 4578$ & CTC2 & $6684 / 6796$ \\
\hline & CTC3 & $5052 / 4025$ & CTC3 & $7551 / 8996$ & CTC3 & $4319 / 4361$ & СTC3 & $6336 / 6618$ \\
\hline & CTC4 & $3600 / 4493$ & CTC4 & $6408 / 7374$ & CTC4 & $3008 / 2990$ & CTC4 & $6105 / 6612$ \\
\hline
\end{tabular}

5 Table S1. Average moisture transport for precipitation (MTP) for each class representing circulation types before and after the 2003 change point 


\begin{tabular}{|c|c|c|c|c|c|c|c|c|}
\hline & CTC & Atlantic & Mode & Pacific & Mode & Siberian & Mode & North America \\
\hline \multirow[t]{4}{*}{ June } & CTC1 & 9.30/5.31 & CTC1 & $6.5 / 6.69$ & CTC1 & $9.38 / 8$ & CTC1 & $7.75 / 4.92$ \\
\hline & СТC2 & $12.67 / 13.61$ & CTC2 & $16.29 / 14.61$ & CTC2 & $10.41 / 9.85$ & CTC2 & $14.71 / 13.54$ \\
\hline & CTC3 & $5.38 / 7.38$ & CTC3 & $4.54 / 6.46$ & CTC3 & $5.62 / 6.77$ & СТC3 & $4.5 / 8.46$ \\
\hline & CTC4 & $2.67 / 3.69$ & CTC4 & $2.67 / 2.23$ & СTC4 & $4.58 / 5.38$ & CTC4 & $3.04 / 3.08$ \\
\hline \multirow[t]{4}{*}{ July } & CTC1 & $14.08 / 9.85$ & CTC1 & $11.17 / 8.62$ & CTC1 & $9.04 / 6.23$ & CTC1 & $10.63 / 7.85$ \\
\hline & CTC2 & $14.17 / 16.23$ & CTC2 & $13.13 / 14.62$ & СTC2 & $6.63 / 7.23$ & CTC2 & $16.83 / 16.31$ \\
\hline & СТC3 & $1.75 / 3.54$ & CTC3 & $3.79 / 5.54$ & СТC3 & $9.54 / 10.38$ & СТC3 & $3 / 6.23$ \\
\hline & CTC4 & $1 / 1.38$ & CTC4 & $2.92 / 2.23$ & CTC4 & $5.79 / 7.15$ & CTC4 & $0.54 / 0.62$ \\
\hline \multirow[t]{4}{*}{ August } & CTC1 & $16.63 / 10.38$ & CTC1 & $10.92 / 8.77$ & CTC1 & $9.83 / 12.54$ & CTC1 & $15.58 / 9.08$ \\
\hline & CTC2 & $11 / 15.62$ & CTC2 & $13.92 / 17.46$ & СТC2 & $8.17 / 10$ & CTC2 & $13 / 15.69$ \\
\hline & СТC3 & $2.45 / 4.24$ & CTC3 & $2.63 / 2.77$ & CTC3 & $7.67 / 4$ & CTC3 & $1.83 / 4.92$ \\
\hline & CTC4 & $0.96 / 0.77$ & CTC4 & $3.54 / 2$ & CTC4 & $5.33 / 4.46$ & CTC4 & $0.58 / 1.31$ \\
\hline \multirow[t]{4}{*}{ Summer } & CTC1 & $40 / 25.50$ & CTC1 & $28.58 / 24.08$ & CTC1 & $28.25 / 26.77$ & CTC1 & $33.96 / 21.84$ \\
\hline & CTC2 & $37.79 / 45.46$ & CTC2 & $43.33 / 46.69$ & CTC2 & $25.21 / 27.08$ & CTC2 & $44.54 / 45.53$ \\
\hline & CTC3 & $9.58 / 15.15$ & CTC3 & $10.96 / 14.77$ & CTC3 & $22.83 / 21.15$ & CTC3 & $9.33 / 19.61$ \\
\hline & CTC4 & $4.62 / 5.85$ & CTC4 & $9.12 / 6.46$ & CTC4 & $15.71 / 17$ & CTC4 & $4.17 / 5$ \\
\hline \multirow[t]{4}{*}{ September } & CTC1 & $11.13 / 16.08$ & CTC1 & $7.83 / 10.46$ & CTC1 & $6.29 / 5.38$ & CTC1 & $6.38 / 8.31$ \\
\hline & CTC2 & $12.67 / 9.08$ & CTC2 & $12.17 / 12.54$ & CTC2 & $12.5 / 13.54$ & CTC2 & $16.67 / 16.54$ \\
\hline & CTC3 & $5.08 / 4.46$ & CTC3 & $7.75 / 5.46$ & СTC3 & $7.21 / 7.84$ & CTC3 & $4.92 / 3.61$ \\
\hline & CTC4 & $1.12 / 0.38$ & CTC4 & $2.25 / 1.54$ & CTC4 & $4.00 / 3.23$ & CTC4 & $2.04 / 1.54$ \\
\hline \multirow[t]{4}{*}{ October } & CTC1 & $11.17 / 8.46$ & CTC1 & $10.33 / 11.08$ & CTC1 & $15.38 / 14.23$ & CTC1 & $8.25 / 5.31$ \\
\hline & CTC2 & $12.00 / 12.84$ & CTC2 & $13.88 / 13.15$ & CTC2 & $11.25 / 12.15$ & CTC2 & $15.75 / 15.23$ \\
\hline & СТC3 & $5.50 / 5.00$ & СТC3 & $4.17 / 2.46$ & СTC3 & $3.67 / 3.85$ & СТC3 & $4.46 / 7.23$ \\
\hline & CTC4 & $2.33 / 4.69$ & CTC4 & $2.62 / 4.31$ & CTC4 & $0.71 / 0.77$ & CTC4 & $2.54 / 3.23$ \\
\hline \multirow[t]{4}{*}{ November } & CTC1 & $10.21 / 11.08$ & CTC1 & $6.71 / 8.08$ & CTC1 & $12.33 / 14.31$ & CTC1 & $7.92 / 8.92$ \\
\hline & CTC2 & $12.37 / 10.38$ & CTC2 & $15.71 / 13.69$ & CTC2 & $13.46 / 12.08$ & CTC2 & $16.67 / 16.84$ \\
\hline & CTC3 & $4.67 / 7.23$ & СТC3 & $2.59 / 5.00$ & CTC3 & $4.00 / 2.77$ & СТC3 & $3.08 / 3.77$ \\
\hline & CTC4 & $2.75 / 1.31$ & CTC4 & $5.00 / 3.23$ & CTC4 & $0.21 / 0.85$ & CTC4 & $2.33 / 0.46$ \\
\hline \multirow[t]{4}{*}{ Autumn } & CTC1 & $32.51 / 35.62$ & CTC1 & $24.87 / 29.62$ & CTC1 & $34.00 / 33.92$ & CTC1 & $22.55 / 22.54$ \\
\hline & CTC2 & $37.04 / 32.26$ & CTC2 & $41.76 / 39.38$ & CTC2 & $37.35 / 37.77$ & CTC2 & $49.09 / 48.61$ \\
\hline & CTC3 & $15.25 / 16.83$ & CTC3 & $14.51 / 12.92$ & CTC3 & $14.88 / 14.46$ & CTC3 & $12.46 / 14.61$ \\
\hline & CTC4 & $6.20 / 6.38$ & CTC4 & $9.87 / 9.08$ & CTC4 & $4.92 / 4.85$ & CTC4 & $6.91 / 5.23$ \\
\hline
\end{tabular}

Table S2. Changes in the frequency of each class (days per month) before and after the CP. Blue/red coloured numbers indicate decreased/increased frequency of the class after the CP. Colours in the CTC cells are related to the order 5 relationship of the 1980-2016 average MTP value for each CTC (in decreasing order: red, orange, green, and blue). 